\title{
La transformation des organisations de soins et du travail médical par le recours à l'information au Québec: une analyse critique
}

Luc Bonneville

\section{OpenEdition}

Journals

Édition électronique

URL : http://journals.openedition.org/communicationorganisation/3291

DOI : 10.4000/communicationorganisation.3291

ISSN : $1775-3546$

Éditeur

Presses universitaires de Bordeaux

Édition imprimée

Date de publication : 2 janvier 2005

Pagination : 201-222

ISSN : 1168-5549

Référence électronique

Luc Bonneville, "La transformation des organisations de soins et du travail médical par le recours à l'information au Québec: une analyse critique », Communication et organisation [En ligne], 26 | 2005, mis en ligne le 19 décembre 2012, consulté le 19 avril 2019. URL : http://journals.openedition.org/ communicationorganisation/3291; DOI : 10.4000/communicationorganisation.3291

Ce document a été généré automatiquement le 19 avril 2019.

(c) Presses universitaires de Bordeaux 


\title{
La transformation des organisations de soins et du travail médical par le recours à l'information au Québec: une analyse critique
}

\author{
Luc Bonneville
}

\section{Introduction}

1 Au Québec, comme dans la plupart des pays industriels avancés, la transformation du système de santé trouve son origine dans un vaste mouvement de réorganisation, de réforme, qui s'est imposé, au sein des instances décisionnelles, à la suite du décret de crise du secteur de la santé. Cette crise, si l'on suit l'histoire de la santé au Québec, a été diagnostiquée et annoncée au début des années quatre-vingts par l'État et le milieu des affaires sur la base de l'évolution d'indicateurs de performance (budgétaires, organisationnels et démographiques) ${ }^{1}$. Parmi ces indicateurs, ce sont ceux révélant la croissance des dépenses publiques de santé qui ont suscité le plus de réactions de la part des décideurs, ceux-ci voyant en cette croissance un obstacle majeur à l'équilibre budgétaire ainsi qu'à la croissance économique dans un contexte marqué par la lutte au déficit. Il fallait donc, selon l'État et le milieu des affaires, s'inspirant des discours antiinterventionnistes de l'État qui prônaient déjà dans les années soixante et soixante-dix la nécessité de rationaliser l'ensemble des dépenses de l'État, réduire considérablement les dépenses publiques de santé. Deux solutions furent alors mises en avant:1) réduire les dépenses de santé assumées par le secteur public ; 2) augmenter la productivité du travail des professionnels de la santé dans les organisations de soins, considérées comme leur " châteaux-forts", pour diminuer les coûts publics des services de soins offerts à la population. Ce que permettra la mise en place du virage ambulatoire informatisé à partir 
du début des années quatre-vingt-dix, tel que nous l'avons, en outre, constaté au cours de nos recherches ${ }^{2}$.

Par ce virage ambulatoire informatisé, l'État voulait mettre en place une nouvelle organisation du travail investit de la capacité d'intensifier le travail des professionnels de la santé. D'où le recours à des mécanismes marchands, le productivisme, pour y parvenir. Entre autres, comme le précise Gadrey, les services hospitaliers, compte tenu qu'ils occupent une part importante des dépenses publiques de santé ${ }^{3}$, représentaient une cible pertinente pour les décideurs.

3 Compte tenu de leur poids dans l'activité et dans les dépenses, les services hospitaliers semblent d'excellents candidats pour la recherche de "gains de productivité», et la plupart des discours sur la maîtrise des dépenses de santé font état de l'existence de "gisements de productivité» qui, dans l'état actuel des choses, resteraient inexploités".

Avec la réorganisation des services publics de soins, on voulait dans ce contexte saisir les opportunités offertes par les technologies de l'information et de la communication (TIC), dont l'État avait d'ailleurs déjà affirmé certaines promesses liées à leur utilisation quotidienne $e^{5}$, pour transformer les façons de faire, les pratiques, des organisations de soins et tout autant des professionnels de la santé, de manière à diminuer les coûts des services de soins assumés par le secteur public. Or, cette façon de concevoir et donc de mettre en place l'informatisation, suivant les objectifs défendus par l'État ${ }^{6}$, a conduit les professionnels de la santé, dans les organisations de soins, à subir la transformation de leur pratique suivant des impératifs d'intensification modelés sur la production industrielle de biens de consommation en série. Voilà ce que nous tenterons de montrer dans cet article, en appuyant notre propos sur des extraits d'entretiens effectués sur le terrain.

\section{Méthodologie}

Dans le but de comprendre l'impact de l'informatisation des organisations de soins et du travail médical au Québec, nous avons mené des entretiens semi-structurés (25) auprès d'acteurs clés impliqués, principalement à titre de décideurs, dans la mise en place du virage ambulatoire informatisé. L'analyse des entretiens nous a permis de comprendre les motifs principaux pour lesquels l'informatisation dans le secteur de la santé a été mis en place, et pourquoi cette informatisation a surtout été orientée vers les organisations de soins avec comme cible principale les professionnels de la santé. L'effort consistait à saisir, comprendre, pourquoi et comment l'informatisation devait représenter un moyen conçu comme crucial - par l'État québécois ${ }^{7}$ - pour transformer les organisations de soins ainsi que le travail médical. En donnant toute la latitude d'expression à nos informateurs, les entretiens semi-structurés nous ont ainsi permis de mettre en lumière les objectifs et buts profonds de l'opération de transformation des organisations de soins et du travail médical par le recours à l'informatisation.

6 Notre population d'enquête a été segmentée en quatre principales catégories d'informateurs : 1) une catégorie de hauts fonctionnaires, à la tête des directions au sein du Ministère de la Santé et des services sociaux du Québec, des régies régionales ou au sein d'organisations de soins, impliqués dans la réorganisation du système de santé autour du virage ambulatoire informatisé; 2) une catégorie de responsables administratifs d'expérimentations ou de projets en télé-médecine à visée clinique au sein 
d'organisations de soins; 3) une catégorie de professionnels de la santé à la tête d'expérimentations ou de projets en télé-médecine à visée clinique ;4) une catégorie de promoteurs de nouvelles technologies, c'est-à-dire de gestionnaires du secteur privé dont le rôle consiste à promouvoir l'utilisation des TIC dans la pratique médicale quotidienne.

\section{L'intensification du travail médical par l'informatisation des organisations de soins}

\section{Restructuration, intensification et surcharge de travail pour les professionnels de la santé oeuvrant dans les organisations de soins}

7 La logique productiviste privilégiée par les hauts fonctionnaires, les responsables de projets et les promoteurs de nouvelles technologies en santé que nous avons interrogé, requérait une restructuration du travail médical dans le but de conduire à des gains de productivité, lesquels devaient contribuer à la diminution des coûts des services publics de soins. Cet objectif fondamental ne pouvait être atteint, pour plusieurs informateursdécideurs interrogés au moment de l'enquête, que par la mise en place de moyens par lesquels le travail des professionnels de la santé pouvait, le pensait-on, s'intensifier. Une intensification qui devait notamment se traduire par une augmentation de la rapidité avec laquelle les patients sont pris en charge, soignés ${ }^{8}$. Or, cette intensification du travail des professionnels de la santé devait inévitablement déboucher sur un alourdissement de la charge quotidienne de travail de ceux-ci. Sur le terrain, dans la quotidienneté du travail médical, cela a voulu dire une augmentation du nombre de tâches que les professionnels de la santé devaient accomplir dans un temps donné, comprimé, dans un contexte où les ressources mises à disposition des organisations de soins étaient de plus en plus restreintes ${ }^{9}$. Pour trois de nos informateurs, professionnels de la santé, il était clair que cela conduisait à une surcharge de leur travail :

Il y a eu une surcharge de travail, il ne faut pas se le cacher. C'est officiel, on ne peut pas se le cacher. La fin de semaine, le centre ambulatoire est fermé. Les gens travaillent donc régulièrement en temps supplémentaire. Nous n'avons jamais refusé quelqu'un [un patient] qui nous était référé, de façon à ce qu'il ne soit pas hospitalisé. Ainsi, puisque les gens [patients] sont toujours acceptés, ils s'ajoutent à notre agenda. On travaille beaucoup plus fort que sur les étages, parce qu'il y a tellement de patients. Beaucoup de patients. [...] Le problème, c'est que trois infirmières ont fait un burn out. [...] Il y a plus de travail qu'il y en avait. On court. [...] Cela faisait partie des engagements du centre ambulatoire, c'est-à-dire d'offrir une alternative à l'hospitalisation. Mais il faut donner le service.

9 Au niveau du module adulte-enfant du centre ambulatoire, il y a eu des burn outs. [...] Les infirmières débutaient leur journée de travail vers 8 heures le matin, pour la terminer entre $17 \mathrm{~h} 30$ et $18 \mathrm{~h} 30$, à tous les jours, du lundi au vendredi. [...] Il y a eu une augmentation de la clientèle en peu de temps. [...] Nous ne retournions pas les patients aux médecins, parce que ceux-ci se sentaient en insécurité. Nous ne retournions donc personne en plus des patients qui s'ajoutaient à nos consultations. Nous n'avions plus de temps. Deux des infirmières terminaient leurs journées de travail très tardivement.

Ces témoignages montrent très clairement que la réorganisation du travail médical dans les organisations de soins a débouché sur une intensification du travail des professionnels 
de la santé, lesquels ont vu leurs tâches quotidiennes s'alourdir de façon considérable comme en témoignent d'ailleurs les problèmes de santé auxquels eux-mêmes ont été confrontés compte tenu de cet alourdissement. Ce phénomène qui prend de plus en plus d'importance dans le secteur de la santé, comme l'évoque la chronique quotidienne des vicissitudes du travail des professionnels de la santé au cours des quinze dernières années, trouve son origine dans le projet d'augmentation de la productivité du travail médical rendue notamment possible par la réorganisation des soins autour des TIC. Tel que nous l'avons indiqué plus haut, cette réorganisation a pour but la maximisation du rendement des professionnels de la santé, tel que l'affirment un membre important de la direction d'un centre hospitalier et un directeur d'un service du génie-biomédical d'un autre centre hospitalier :

Notre philosophie consistait à donner au médecin un accès à l'information. Un accès facile, rapide et intégré, afin qu'il puisse maximiser son potentiel de médecin à l'hôpital avec toutes les connaissances dont il dispose. [...] Les professionnels doivent travailler en équipe autour de l'information pour maximiser leur productivité vis-à-vis du patient. [...] Ensuite, ils vont pouvoir faire en sorte que nous ayons la meilleure qualité des soins au meilleur coût [...]. Avec cette technologie, on pourrait facilement permettre au médecin de compléter un dossier à distance au moment qui lui convient, c'est-à-dire que ce soit au bureau, à l'hôpital, voire même de chez lui. C'est un avantage énorme.

Ces extraits d'entretiens menés auprès de décideurs et de gestionnaires indiquent très nettement que l'objectif premier de la réorganisation du système de santé, par l'informatisation des organisations de soins, est l'augmentation de la productivité des différents professionnels de la santé, en grande partie des médecins, dans le cadre des services publics de soins. On veut qu'ils fassent plus, plus rapidement, qu'ils allongent même leur journée de travail « compléter un dossier à distance [...] même chez lui ». Le travail en réseau, dans un tel contexte, est considéré comme un instrument d'optimisation du rendement des professionnels de la santé «maximiser son potentiel de médecin à l'hôpital [...] leur productivité vis-à-vis du patient ». Ce qui est vu comme bénéfique pour les organisations de soins « c'est un avantage énorme ».

Par ailleurs, cette augmentation de la productivité, spécialement du travail des médecins, opère sur la base d'une dynamique grâce à laquelle on confère aux professionnels plus nombreux, mais qui coûtent moins cher, notamment les infirmières et infirmiers, un maximum de tâches dans un temps donné. Ce qui coûte moins cher à l'acte, au geste, du fait que les médecins et les infirmières peuvent traiter plus de patients dans un temps donné pour un coût donné qu'on espère le plus bas possible. Les propos d'un directeur des services professionnels d'un centre hospitalier sont particulièrement illustratifs à ce sujet :

14 Il y a une résistance chez certains médecins. Ceux-ci se demandent pourquoi ils devraient suivre 25 à 30 patients de plus à distance, alors qu'ils n'ont aucune rémunération supplémentaire. Ils le font de temps à autre, sauf que les volumes [d'activités] ont tendance à augmenter. Il est légitime qu'ils se posent des questions. Par exemple si les patients étaient hospitalisés plutôt qu'à domicile les médecins seraient rémunérés, alors qu'avec le système de télésurveillance ils ne le sont pas. Nous avons des médecins volontaires, mais il y a une limite à ce bénévolat, à ce volontariat. [...Par ailleurs...] on s'attend à ce que l'infirmière ait un rôle beaucoup plus large et beaucoup plus riche [ce qui veut dire plus productif], c'est-à-dire un rôle clinique ou un rôle professionnel. Je dirais que nous avons tendance à nous diriger vers la pratique avancée en soins 
infirmiers. [...] C'est ce que les Américains et Anglophones nomment la nurse practitionner, c'est-à-dire une infirmière qui se rapproche du médecin dans son rôle. On voudrait donc que les infirmières aient une plus grande marge de manoeuvre, ne serait-ce que pour dire au patient quoi faire en pouvant prescrire par exemple un électrocardiogramme, une radiographie du poumon, etc., à l'intérieur de protocoles. Les tâches des infirmières consisteraient à recevoir de l'information, à l'interpréter cliniquement, à faire des interventions sur des conseils pratiques réguliers, voire même qu'elles aillent au-delà de ces tâches en pratique avancée un jour, même au niveau de la prescription de certaines choses.

Si une telle restructuration entre les métiers et les professions conduit à une augmentation de la productivité du travail des médecins, à partir du moment où leur nombre sera proportionnellement moins grand par rapport à la quantité des interventions cliniques nécessaires pour traiter les patients à la suite de la substitution de leur travail vivant par un autre, elle conduit également à un accroissement important des tâches principalement dévolues aux infirmières et infirmiers, ce qu'on conçoit ici de manière très favorable " un rôle beaucoup plus large et beaucoup plus riche ", mais qui masque une intensification telle du travail qu'elle lui donne l'allure d'un amalgame confusionnel, comme cet extrait d'entrevue conduite auprès d'un directeur de projet expérimental le montre :

Lorsqu'un intervenant est chez Madame A, il va pouvoir gérer toutes ses affaires. Il a accès à son dossier et à ses informations. [...] Lorsqu'il sort de chez Madame $\mathrm{A}$, son intervention est terminée. S'il doit téléphoner au médecin, il le fait chez Madame A. Ils [les intervenants de santé] vont donc offrir le même service chez les 7 clients qu'ils rencontrent. Mais au lieu de visiter 7 clients le matin et de faire de la paperasse l'aprèsmidi, ils vont faire tout de $\mathrm{A}$ à $\mathrm{Z}$ chez Madame $\mathrm{A}$, de $\mathrm{A}$ à $\mathrm{Z}$ chez Madame $\mathrm{B}$ aussi, etc. Les intervenants sont donc sur la route et chez les clients toute la journée.

Dans cet extrait, on pose les avantages de l'informatisation, des TIC, par rapport à leur capacité de contraindre les professionnels de la santé, dans ce cas-ci des infirmières et infirmiers, à accomplir un plus grand nombre de tâches dans un temps donné « ils vont faire tout de $\mathrm{A}$ à $\mathrm{Z}$ chez Madame $\mathrm{A}$, de $\mathrm{A}$ à $\mathrm{Z}$ chez Madame $\mathrm{B}$ aussi, etc. ». Dans ce cas, c'est le rapport au temps qui est modifié par une accélération de la prise en charge clinique de la maladie et des patients. Le but de l'opération consiste à augmenter structurellement la productivité du travail médical non seulement au niveau de chaque travailleur spécifique, individuel, mais aussi pour chaque catégorie de professionnels ou de métier et par ailleurs au niveau systémique même, en transformant l'équilibre entre les composantes du système. Cette conception de l'augmentation de la productivité, inspirée des principes tayloristes d'organisation industrielle du travai ${ }^{10}$, invite également à la remise en cause de l'autorité légitime des professionnels de la santé ainsi que de leur autonomie et pouvoir dans l'organisation générale des soins, en reconfigurant le travail médical suivant des standards, normes, de productivité adaptés à cette conception tayloriste du travail. Cependant, pour y parvenir, il fallait mettre en place des procédures normalisées d'accomplissement du travail médical. D'où la standardisation des tâches et des actes cliniques qui en a résulté. 


\section{Standardiser dans les organisations de soins le travail médical pour le rendre plus productif}

La restructuration du travail médical dont il vient d'être question passe par une standardisation des tâches, qui consiste à reconfigurer le travail des professionnels de la santé autour d'une norme, d'un rapport quantum-temps. Il sera défini a priori en fonction des objectifs assignés à la réorganisation. Elle constitue l'une des composantes fondamentales de la division technique du travail industriel qui exige que l'organisation du travail soit segmenté, décomposé, en différentes parties afin de les mesurer, des les chronométrer, pour identifier le nombre maximal de choses, ici de traitements, qui peuvent être produits le plus rapidement possible ${ }^{11}$. Elle permet donc de rationaliser, d'objectiver, la fixation d'un optimum d'utilisation des ressources dans le cadre même des restrictions budgétaires importantes ${ }^{12}$.

C'est cette logique productiviste qui a présidé à l'élaboration et à la mise en place de plusieurs projets d'informatisation dans le secteur de la santé au Québec, dont un concernant les salles d'urgences, et pour lequel un membre de l'Ordre des infirmières et infirmiers du Québec (OIIQ) a insisté sur le problème auquel le chronométrage des actes conduisait :

Le logiciel indique exactement le temps consacré à la consultation. Le médecin ne veut pas voir cela. Vous savez, il y a un chronomètre dans cette technologie. C'est l'enfer. [...] Un médecin m'a déjà dit qu'il n'en revenait pas que le système puisse le contrôler avec son chronomètre. Il n'était pas question qu'il utilise cette technologie.

21 La standardisation et la contraction du rapport au temps, qui sont objectivées par le chronomètre, se renvoient donc l'une à l'autre dans une reconfiguration de l'organisation du travail qui alourdit et intensifie le travail des professionnels de la santé. Or, tel que nous l'avons constaté, ce phénomène a suscité une résistance certaine de la part des professionnels de la santé. Cependant, cette standardisation - intensification est considérée comme un élément central, incontournable, de la réorganisation par ceux qui la conçoivent comme nécessaire et qui sont chargés de la mettre en place, tel que le soulignent ces propos d'un haut fonctionnaire au Ministère de la Santé et des services sociaux et d'un directeur des services professionnels dans un centre hospitalier :

Nous avons implanté d'autres systèmes tels que les infosantés. Il y a des protocoles standardisés pour tout le monde, grâce à l'informatisation. On dit la même chose à tous les patients, partout dans la province, face à un même problème de santé.

[...] Puisque nous voulions utiliser nos ressources de façon appropriée [ce qui veut dire de façon optimum], il fallait que nos médecins aient des pratiques un peu plus standardisées. [...] On met nos médecins en "concurrence» les uns avec les autres, pour dire par exemple à un médecin que son collègue hospitalise ses patients moins ou plus longtemps que lui. Par exemple, on pouvait se demander pourquoi notre taux d'hospitalisation pour la même population en cardiologie était plus ou moins élevé par rapport à d'autres établissements. On confrontait les médecins dans leurs décisions cliniques. La technologie dont je vous parle participe à cette dynamique.

Cette nécessité explicitée de standardiser le travail des professionnels de la santé, par les TIC, répond à des impératifs industriels d'organisation de la production, comme si les services de soins étaient des objets, des produits, qu'on peut accomplir plus rapidement, 
en plus grande quantité, avec des ressources les plus restreintes possible ${ }^{13}$. Ceci dans le but d'identifier un rendement optimum idéal qui se conjugue avec la diminution des coûts des services de soins. Dans ce contexte, la quête de gains de productivité devient non seulement le moyen pour augmenter le rendement global du système de santé, mais une fin en soi justifiée par l'impérativité des économies croissantes. C'est pourquoi les TIC sont conçues, par nos informateurs-décideurs, comme le moyen par excellence pour introduire une dynamique productiviste en santé qui s'inspire précisément, comme nous l'avons indiqué plus haut, de l'organisation industrielle tayloriste du travail ${ }^{14}$. En témoignent les propos suivants d'un membre de la direction d'un centre hospitalier :

Il faut réviser les processus, faire un reengineering. [...] Il faut mettre en place la technique du material resource planning (MRP) dans le secteur manufacturier, où le processus de production d'une automobile est extrêmement standardisé. Même si vous prenez deux travailleurs qui ne travaillent pas au même endroit et qui ne parlent pas la même langue, l'automobile va être produite de façon identique. Aujourd'hui, dans une organisation qui ne standardise pas ses processus, deux personnes différentes vont produire deux ratios différents de patates. L'informatisation permet de faire cette standardisation. Pour gérer ce processus une fois mis en place, nous devions avoir de l'information afin de faire une analyse de la performance avec des indicateurs clés, les délais, les ressources, etc. Cela permet de mettre en œuvre des benchmark ou des objectifs clés, donc de pouvoir convenir d'un temps pour chaque processus. [...] On peut arriver à gérer cela, par exemple pour l'urgence. [...] L'informatisation permet ainsi de mettre en place toute une infrastructure de gestion des opérations. [...] Le fait de gérer chaque processus à l'hôpital va nous permettre, non seulement de produire des services au meilleur coût, mais de prévoir nos coûts et de s'assurer que ceux-ci ne varient que très peu. Par exemple, aujourd'hui, pour deux patientes du même âge qui entrent dans deux hôpitaux différents à la suite d'une fracture de la hanche en ski, nous n'avons aucune idée des coûts que cela va occasionner, compte tenu des variations trop grandes qui se présentent. Nous n'avons aucune garantie sur le coût pour chaque patiente, à savoir laquelle des deux va être hospitalisée plus longtemps et laquelle va coûter plus cher. Nous voulons donc en arriver à réduire la variation des coûts.

Ici, le lien qui est fait entre l'industrie automobile et les services de soins nous apparait clairement significatif de la conception du travail médical qui prévaut chez les décideurs. On pense que les services de soins peuvent se soumettre à l'organisation du travail caractéristique du milieu industriel, comme s'ils étaient des choses qui peuvent être produites et reproduites en série, comme dans le cas d'une chaine de montage où les coûts de chaque facteur de production est déterminé en amont. Les TIC doivent donc être utilisées, suivant cette conception, comme instrument d'optimisation du mode de fonctionnement des organisations de soins. On oublie cependant que la finalité même de ces dernières est d'offrir des services de la meilleure qualité possible, d'où l'intérêt d'une prise en compte des conditions du travail des professionnels de la santé. Pourtant, le témoignage qui suit de la part du même membre de la direction d'un centre hospitalier, indique clairement que les organisations sont considérées comme des entreprises, voire des manufactures, qui produisent en série, de façon homogène et standardisée, des objets de consommation, ici des services de soins. D'où la possibilité d'augmenter la productivité de l'ensemble du " processus de production » permettant d'offrir le plus de soins possible, avec les instruments adéquats que représentent les TIC. 

de l'innovation, pour ultimement en arriver un jour où l'on pourra peut-être gérer une partie de l'hôpital comme une manufacture. Il y a des choses qui sont contrôlables et prévisibles, d'autres qui ne le sont pas. Par exemple, les visites à l'urgence ne sont pas prévisibles. Il peut aujourd'hui y en avoir 30 ou 200. Par contre, quand le patient arrive à l'urgence et qu'on a un diagnostic, on peut assez bien prévoir son cheminement dans l'hôpital. Il faut donc en tenir compte. Présentement, on ne peut rien planifier, parce qu'on ne peut rien prévoir. Nous contrôlons à peu près rien. L'informatisation va permettre justement de contrôler tous ces éléments. [...] En informatisant, on rend l'information disponible tout en standardisant. On réduit ainsi les variations et on met en place des indicateurs clés de performance pour évaluer le processus. On tente de vérifier les coûts.

Dans cet extrait, il est clairement affirmé que l'informatisation des services de soins avait pour objectif fondamental la réduction des coûts du travail des professionnels de la santé. Il s'agit ici de contrôler les coûts de tous les facteurs de production mis à contribution dans les organisations de soins considérées comme des manufactures, en partant du principe que le «meilleur» service de soins est celui qui coûte le moins, c'est-à-dire dont le coût de production est le plus bas possible. C'est ce qu'affirme un haut fonctionnaire du Ministère de la Santé et des services sociaux du Québec (MSSS) dans ces mots :

Ce dont je vous ai parlé, c'est de la performance reliée aux ressources humaines. La performance reliée aux aspects cliniques doit passer par ce qu'on nomme le coût de revient. [...] Il faut être capable d'associer une maladie X, par exemple un problème d'hépatite ou de cancer, avec des standards établis. Par exemple, nous devons nous demander pourquoi un établissement dépasse le standard, alors qu'un autre ne le dépasse pas. Il faut donc établir le coût réel d'une pathologie, d'un acte médical. Je pense que Monsieur Legault aura besoin d'informations pour mettre en place les contrats de performance, de façon à ce que les établissements puissent être comparés les uns aux autres. Ne serait-ce que pour pouvoir dire, par exemple, que dans tel hôpital le séjour hospitalier pour une fracture de la hanche est de X jours, alors que la moyenne provinciale est de cinq jours. Pourquoi dans tel établissement pour une fracture de la hanche, il y a eu huit rayons X et quatre dans un autre? Que se passe-t-il ? Il faut donc avoir les éléments pour être capable de comparer les pratiques professionnelles et les performances hospitalières. Pour reprendre l'exemple des rayons $\mathrm{X}$, on veut savoir combien ils coûtent dans tel ou tel établissement, pour un même type de clientèle. Combien coûte un repas dans tel ou tel hôpital ? Nous aurons besoin de telles données éventuellement. Outre la circulation de telles données, j'ai besoin d'applications pour les gérer, pour être capable [...] d'établir un contrat de performance. [...] Pour moi, c'est un des éléments importants. Si j'étais directeur général d'un hôpital, il faudrait qu'on me donne les moyens d'être capable de gérer cette organisation.

Dans ce témoignage, nous constatons que la standardisation du travail des professionnels de la santé se conjugue avec la possibilité-nécessité d'identifier, ou de déterminer, une norme objective abstraite et arbitraire de "production" qu'on prévoyait imposer à l'ensemble des organisations de soins et du système de santé. Il fallait contraindre les professionnels de la santé à traiter leurs patients suivant des procédures et protocoles prédéterminés et programmés dans les TIC. Il n'est donc pas étonnant d'avoir constaté, suivant les objectifs assignés au virage ambulatoire informatisé ${ }^{15}$, une exigence forte

Communication et organisation, 26 | 2012 
envers les professionnels de la santé dont on veut qu'ils fassent "plus », plus rapidement et avec « moins » de ressources à leur disposition.

\section{Le leitmotiv des organisations (informatisées) de soins : faire " plus » avec « moins » de ressources}

Pour plusieurs de nos informateurs-dédideurs, la standardisation du travail des professionnels de la santé était devenue " nécessaire », « inéluctable » voire " urgente » pour augmenter la productivité des organisations de soins, dont le budget de fonctionnement est désormais fixe et impérativement indépassable, suivant la Loi antidéficit en santé dont nous avons souligné l'importance plus haut, tel que l'affirme un chef de projet expérimental à la Régie de l'assurance-maladie du Québec :

Il faut comprendre que le système [sociosanitaire] fonctionne maintenant sur la base d'une enveloppe budgétaire fermée.

Voilà pourquoi il fallait, pour plusieurs de nos informateurs-décideurs, augmenter la productivité du travail des professionnels de la santé de façon à ce que les organisations puissent faire face à l'augmentation croissante des besoins de soins ${ }^{16}$. Il fallait ainsi contraindre ces dernières à prendre en charge le plus grand nombre de patients donnés dans un temps donné, de plus en plus court. C'est précisément ce qu'on affirmé un promoteur de nouvelles technologies, deux responsables médicaux d'un centre ambulatoire régional, deux hauts fonctionnaires du Ministère de la Santé et des services sociaux du Québec (MSSS) travaillant dans deux directions différentes, un radiologue en chef d'un projet expérimental dans un centre hospitalier et un promoteur de nouvelles technologies :

Une infirmière à un poste de télésurveillance peut surveiller entre 75 et 100 patients de façon simultanée, ce qui n'est pas possible à l'intérieur d'un département. Il faut donc concentrer la demande pour la traiter de façon centrale ainsi qu'offrir des services au centre hospitalier et aux établissements environnants qui n'ont pas nécessairement la présence d'un intervenant pour surveiller les patients

Ici, nous traitons beaucoup plus de patients. C'est un objectif prioritaire de soigner un plus grand nombre de patients. [...] Il y a des interventions de groupe qui nous permettent de prendre plus de patients à la fois. On peut quintupler le nombre de patients pour le même temps.

L'organisation interne [du centre ambulatoire] permet de traiter plus de patients, parce que les gens qui sont ici sont des cas lourds souvent très malades. Lorsque nous les voyons, ils ont déjà eu des prises de sang par l'infirmière, qui a aussi pris connaissance de leurs signes vitaux. [...] Les patients doivent toujours être prêts à être examinés par les médecins. [...] Cela nous permet de traiter plus de patients, c'est clair.

On va traiter le plus de patients possible avec le même argent. C'est ce qui va se passer.

Avec le virage ambulatoire, nous avons sorti certains patients des établissements, parce que les résultats de leurs tests sont produits rapidement. Nous avons donc augmenté l'efficience, l'efficacité probablement, mais les lits continuent de se remplir quand même. Cependant, plutôt que de traiter 1 patient par lot en 5 jours, tu en traites 2 dans la même période. Ce qui fait qu'on augmente la rentabilité, il va sans dire. 

deux fois à tous les deux semaines, pour s'assurer que tel patient est bien suivi. Ces réunions étaient nécessaires puisque les intervenants ne sont pas dans une dynamique de partage de l'information. Or, si les intervenants travaillaient avec la même information et en collégialité, ils n'auraient pas besoin de se réunir. Ils verraient donc cinq patients de plus. professionnels de la santé, qui passe par une augmentation du nombre de patients traités dans un temps donné, trouve sa pertinence, selon deux des médecins que nous avons rencontrés, qui sont aussi directeurs des services professionnels dans des centres hospitaliers, par la pénurie en ressources humaines qui affecte le système québécois de santé :

Je vois beaucoup d'effets secondaires au niveau de la gestion du personnel. Par exemple, j'ai la possibilité d'avoir un ergothérapeute 3 jours par semaines, puisque je n'ai pas de budgets pour en avoir un 5 jours par semaine. Or, je ne suis pas capable de recruter un bon ergothérapeute pour seulement 3 jours par semaine. Qui va s'isoler dans cette ville pour y travailler à temps partiel ? [...] Par contre, un peu plus loin dans d'autres villages, ils ont besoin d'un ergothérapeute, mais seulement pour 2 jours par semaine. On pourrait donc offrir un poste de 5 jours par semaine, dont 2 jours en téléconsultation. Cela peut se faire. était préférable d'utiliser le personnel déjà en poste pour maximiser leur productivité, par une accélération de leurs gestes et actes quotidiens, afin d'augmenter la quantité de patients traités dans un temps donné. Ainsi se comprend la nécessité affirmée d'informatiser les organisations de soins de manière à solutionner, à résoudre, entre autres, ce que nos informateurs-décideurs considèrent comme une duplication des tâches des professionnels de la santé qui repose, selon eux, sur une mauvaise gestion de l'information au sein des organisations de soins, comme le laissent entendre ces propos d'un responsable administratif d'un projet expérimental dans une régie régionale, de deux hauts fonctionnaires du Ministère de la Santé et des services sociaux du Québec (MSSS) travaillant dans deux directions différentes, d'un promoteur de nouvelles technologies, d'un responsable médical d'un projet régional en télé-médecine et d'un haut fonctionnaire à la Régie de l'assurance-maladie du Québec (RAMQ) :

Les employés n'ont plus besoin d'aller à leur bureau. Ils sont en mode électronique, plutôt qu'en mode papier. [...] La grosse différence par rapport à l'organisation du travail en 
mode papier se situe au niveau de la duplication d'informations. En effet, les travailleurs ne sont plus obligés de faire de la duplication dans leur journée. La nouvelle organisation [des services de soins] devrait éliminer cette duplication de l'information. Ce sera un gros gain pour les intervenants. [...] L'information sera saisie une fois pour toutes. [...] Ils ont même l'impression qu'à long terme, ils pourraient avoir un gain d'efficience dans le nombre de clients qu'ils pourraient rencontrer par jour, à cause de la réduction de la duplication.

Par exemple, avec la carte à puce, le fait pour le médecin d'obtenir plus rapidement les derniers résultats de laboratoire d'un patient procurerait des économies potentielles. [...] Cela éviterait des duplications de tests, de rayons X, de tests de laboratoires, etc. Cela représente un coût réel.

Sans technologies de l'information, personne ne sait ce que l'autre a fait. Le personnel soignant a donc tendance à recommencer certaines choses. Cela coûte très, très cher. On traite du monde pour rien, parce qu'on recommence ce que l'autre a fait. C'est structurellement et culturellement comme cela.

Le bénéfice pour l'établissement est le suivant. Si toutes les informations de l'imagerie médicale ou des laboratoires sont mises à disposition par le biais d'un même médium, le médecin n'a plus besoin d'envoyer, à nouveau, tel patient passer un autre examen. Avec la technologie, il a déjà les informations pertinentes concernant tel patient. C'est une assistance au diagnostic beaucoup plus rapide. [...] Le médecin n'a pas à faire répéter au patient l'information.

Nous cherchions à mettre à la disposition des médecins de l'information en temps réel sur un client. [...] Faire en sorte d'éviter la répétition d'évaluations. [...] Éviter de répéter les questions au client ou à sa famille, parce que le médecin a déjà l'information.

[Les TIC permettent] d'éviter de recommencer des tests identiques. [...] Quand une chose a été faite et bien faite, pourquoi la refaire ? Cela occasionne des coûts énormes.

Pour nos informateurs-décideurs, cette nécessité d'intensifier et de standardiser le travail des professionnels de la santé afin d'éviter, notamment, les duplications - ce qui permettra notamment d'accélérer la prise de décision clinique dans le but de pouvoir traiter plus de patients dans un temps donné - repose encore une fois sur un objectif de diminution des coûts des services de soins. Il est cependant important de noter qu'il en est ainsi pour nos informateurs-décideurs, alors que nos informateurs professionnels de la santé pensent que le fait d'éviter une certaine duplication pourra conduire à une amélioration de la qualité du travail des professionnels de la santé. Sur le terrain, on constate au-delà de ces considérations que la réorganisation du système de santé qui passe entre autres par l'informatisation des organisations de soins, que la logique qui prévaut est celle d'un productivisme dont l'effet est de diminuer, en théorie ${ }^{17}$, les coûts du travail médical et non d'améliorer la qualité du travail des professionnels de la santé.

\section{Conclusion : l'informatisation comme instrument clé de la rationalisation des pratiques médicales dans les organisations de soins}

Comme nous l'avons montré tout au long de cet article, l'informatisation des organisations a voulu dire une intensification importante du travail médical fondée sur 
les principes tayloristes d'organisation du travail industriel. Cette intensification se pose par rapport à une augmentation de la charge de travail des professionnels de la santé, à partir du moment où l'objectif central consistait à augmenter leur cadence de travail par la réalisation d'un plus grand nombre de tâches, de gestes et d'actes quotidiens dans un temps le plus restreint possible. Il s'agit donc pour les décideurs de saisir les opportunités offertes par les TIC pour augmenter la productivité du travail médical, des professionnels de la santé dans les organisations de soins, de façon à réduire les coûts des services de soins assumés par le secteur public. Une productivité qui, tel que nous l'avons vu, se conjugue avec la contrainte pour les professionnels de la santé de devoir traiter le plus de patients possible dans un temps comprimé et avec des ressources moindre ${ }^{18}$. Pour les décideurs, il s'agissait d'imposer la prééminence de la raison économique sur la raison clinique, l'organisation industrielle tayloriste du travail symbolisant ici les fondements des nouveaux services de soins offerts. Or, nous avons constaté que cette intensification du travail des professionnels de la santé a débouché sur un alourdissement considérable des tâches de ceux-ci, dont le travail a été réorganisé par ailleurs autour de normes et de procédures conçues comme plus productives par ceux qui décident du sens de l'informatisation des organisations de soins.

Dans cette logique productiviste accompagnant l'informatisation des organisations de soins, on considère ainsi que le service de soins comme un produit, une chose, détachés et distincte des acteurs qui y sont pourtant directement impliqués, soit à titre de prestataires de soins soit comme patients. Nous pouvons penser que cette augmentation de la productivité du travail des professionnels de la santé demeure hautement problématique, essentiellement parce qu'on occulte ainsi la dimension centrale d'un service $^{19}$, la relation humaine que la standardisation à des fins productivistes et économiques « secondarise » au nom de la rationalisation.

Ainsi, dans la logique d'implantation privilégiée par l'État et les décideurs locaux, ces considérations liées à la spécificité, à la nature même, des services de soins n'est pas le souci premier; au contraire, tout n'a de sens que par la réduction des coûts publics des services de soins. En oubliant la primauté fondamentale de cette logique, la nouvelle organisation des soins tend à induire une dégradation des conditions du travail médical, à effriter la qualité des soins. On peut donc penser qu'au contraire d'aboutir à des économies, cette manière productiviste de réorganiser les soins par les TIC conduira, du moins à terme, à une augmentation des dépenses de santé, justement du fait de cet effritement ${ }^{20}$. Au centre de cette problématique se situent de nouvelles contraintes imposées à la pratique des professionnels de la santé qui ont insisté, dans leurs témoignages, sur l'idée selon laquelle l'informatisation de leur pratique, suivant les objectifs prédéterminés par les décideurs, occasionnait plusieurs problèmes quant à la possibilité d'améliorer les conditions quotidiennes de leur travail.

On peut finalement penser qu'en réduisant les bienfaits de l'informatisation spécifiquement, voire uniquement, à des considérations de rendement et de productivité, les décideurs mettent en péril le fonctionnement global des organisations de soins dont la survie peut être compromise ${ }^{21}$. Dans un tel contexte, nous sommes d'accord avec AndréPierre Contandriopoulos pour dire que la réduction des coûts des services de soins en soi n'a aucun sens, aucune justification ${ }^{22}$, parce que l'essence de la relation de soins médicaux, des actes de soigner et de se faire soigner, réside dans la relation d'usage même et non dans sa représentation en termes de coûts. 


\section{BIBLIOGRAPHIE}

Association des hôpitaux du Québec, [Page consultée le 29 septembre 2002], «L'augmentation de la demande de services est responsable des difficultés budgétaires des hôpitaux », [En ligne], Adresse URL : http://www.ahq.org/info/nouvelles/presse/c060700.html).

Attewell P., «L'informatique et le paradoxe de productivité », Le communicateur, no. 17, 1992, p. 1-27.

Bonneville L., « La mise en place du virage ambulatoire informatisé comme solution à la crise de productivité du système de santé au Québec (1975 à 2000) », Thèse de doctorat en sociologie, Montréal, Université du Québec à Montréal, 2003.

Comité consultatif sur l'autoroute de l'information, Inforoute Québec - Plan d'action pour la mise en cuvre de l'autoroute de l'information, Québec, CCAI, juillet 1995, 53 p.

Contandriopoulos A.-P., «Économie du système de santé », in : Traité d'anthropologie médicale. L'institution de la santé et de la maladie. Sainte-Foy, Presses universitaire du Québec; Québec, Institut québécois de recherche sur la culture ; Lyon, Presses universitaire de Lyon, 1985.

Carré D., Lacroix J.-G., (dir), La santé et les autoroutes de l'information. La greffe informatique. Paris, L'Harmattan, 2001.

Coriat B., L'atelier et le chronomètre. Paris, C. Bourgois, 1979.

Gadrey J., Services : la productivité en question. Paris, Desclée de Brouwer, 1996.

Gadrey J., L'économie des services. Paris, Éditions La Découverte, 1992.

Institut canadien d'information sur la santé, Tendances des dépenses nationales de la santé, 1975 à 2000, ICIS, Ottawa, 2001

Lacroix J.-G., « Le sens sociopolitique de la façon de construire les autoroutes de l'information dans le secteur de la santé ", in : Actes du colloque Globalisme et pluralisme. Montréal, 24-27 avril 2002, Québec : Presses de l'Université Laval, 2003, p. 117-136.

Lamarche F et Doré M., « L'organisation du travail et les travailleurs ", in : La crise et les travailleurs. Montréal, co-édition Faculté de l'éducation permanente de l'Université de Montréal et Centrale de l'enseignement du Québec, 1980.

Ministère de la Santé et des services sociaux - Direction des ressources informationnelles, Les orientations technologiques du réseau sociosanitaire, Orientations en télécommunication pour le secteur sociosanitaire, Québec, MSSS, novembre 1996.

Ministère de la Santé et des services sociaux - Direction des ressources informationnelles, Les orientations technologiques du réseau sociosanitaire, Guide pour la mise en œuvre, Québec, MSSS, novembre 1996.

Ministère de la Santé et des services sociaux, Direction générale de l'administration et des immobilisations et Direction générale des ressources informationnelles, Prendre l'autoroute de l'information... Les prémisses du plan d'action pour le secteur sociosanitaire, document de travail, Québec, MSSS, octobre 1995 
Ministère de la Santé et des services sociaux, L'autoroute de l'information, un levier essentiel à la transformation du système sociosanitaire - Plan d'action intégré, Québec, MSSS, mai 1997.

Nusbaumer J., Les services. Nouvelle donne de l'économie. Paris, Economica, 1984.

Rallet A., « L'efficacité des technologies de l'information et de la communication à l'étape des réseaux ", in : Monnoyer-Longé Marie-Christine (sous la direction), L'entreprise et l'outil informationnel, Paris, Montréal, L'Harmattan, 1997.

Sicotte C., Champagne F., Contandriopoulos A.-P., «La performance organisationnelle des organismes publics de santé », in : Ruptures, revue transdisciplinaire en santé, vol. 6, no. 1, 1999, p. 34-46.

Taylor F.-W., Principes d'organisation scientifique des usines. Paris, Dunod, 1911.

\section{NOTES}

1. Carré Dominique et Lacroix Jean-Guy (dir.), La santé et les autoroutes de l'information. La greffe informatique, Paris, L'Harmattan, 2001, chapitre 1.

2. Bonneville Luc, « La mise en place du virage ambulatoire informatisé comme solution à la crise de productivité du système de santé au Québec (1975 à 2000) ", Thèse de doctorat en sociologie, Montréal, Université du Québec à Montréal, 2003.

3. Voir Institut canadien d'information sur la santé, Tendances des dépenses nationales de santé, 1975 à 2000, ICIS, Ottawa, 2001.

4. Gadrey Jean, L'économie des services, Paris, Éditions La Découverte, 1992, p. 77.

5. Voir, entre autres, Ministère de la Santé et des services sociaux - Direction des ressources informationnelles, Les orientations technologiques du réseau sociosanitaire, Guide pour la mise en œuvre, Québec, MSSS, novembre 1996, p. 8-9 ; Ministère de la Santé et des services sociaux Direction des ressources informationnelles, Les orientations technologiques du réseau sociosanitaire, Orientations en télécommunication pour le secteur sociosanitaire, Québec, MSSS, 1996, p. 25 ; et Ministère de la Santé et des services sociaux, L'autoroute de l'information, un levier essentiel à la transformation du système sociosanitaire - Plan d'action intégré, Québec, MSSS, mai 1997, p. 53.

6. Bonneville Luc, ouvrage cité, chapitre 4.

7. Bonneville Luc, ouvrage cité, chapitre 4.

8. Bonneville Luc, ouvrage cité, chapitre 5.

9. Telle était d'ailleurs l'annonce officielle qui a été faite par l'État québécois dans plusieurs documents officiels. Voir, notamment, Comité consultatif sur l'autoroute de l'information, Inforoute Québec - Plan d'action pour la mise en ceuvre de l'autoroute de l'information, Québec, CCAI, 1995, p. 17 ; et Ministère de la Santé et des services sociaux - Direction des ressources informationnelles, Les orientations technologiques du réseau sociosanitaire, Orientations en télécommunication pour le secteur sociosanitaire, ouvrage cité, p. 5.

10. Taylor Frederic Winslow, Principes d'organisation scientifique des usines, Paris, Dunod, 1911.

11. Voir, entre autres, Coriat Benjamin, L'atelier et le chronomètre, Paris, C. Bourgois, 1979.

12. Il est ici nécessaire de rappeler que le Gouvernement du Québec a déposé, en juin 2000, une loi sur l'équilibre budgétaire, la Loi 107, qui interdit de façon intransigeante aux organisations de soins de faire des déficits, même dans un contexte de diminution des dépenses publiques de santé. Suivant cette loi, les organisations de soins reçoivent de la part de l'État une enveloppe budgétaire fermée que les gestionnaires doivent respecter sous peine de voir leur établissement mis en tutelle par le Ministère de la Santé et des services sociaux.

13. Ce qui, selon plusieurs chercheurs en économie et sociologie des services, pose d'énormes problèmes en termes de satisfaction de la finalité même des services publics de soins. À ce sujet, 
voir Nusbaumer Jacques, Les services. Nouvelle donne de l'économie, Paris, Economica, 1984 ; et Gadrey Jean, Services : la productivité en question, Paris, Desclée de Brouwer, 1996.

14. Cela avait d'ailleurs été déjà pressenti en 1980 à la suite de l'introduction de l'informatique dans l'organisation du travail médical. Voir à ce propos Lamarche F. et Doré M., «L'organisation du travail et les travailleurs ", dans La crise et les travailleurs, Montréal, co-édition Faculté de l'éducation permanente de l'Université de Montréal et Centrale de l'enseignement du Québec, 1980, p. 83-91.

15. C'est-à-dire des objectifs essentiellement économiques et financiers (Bonneville Luc, ouvrage cité).

16. Mentionnons quelques chiffres soulevés par l'Association des hôpitaux du Québec (AHQ) au sujet de cette augmentation de la demande au sein des établissements: "Dans les salles d'urgence des hôpitaux de Montréal durant les deux dernières années [donc pour 1998 et 1999], le nombre de patients arrivés en ambulance au [sic] augmenté de 19,2\% depuis deux ans (21 808); le nombre de patients admis a quant à lui augmenté de 11823 (hausse de 6,8 \%); dans la région de Québec, le nombre de patients admis a augmenté systématiquement durant les quatre dernières années [donc de 1995 à 1999]. En hémato-oncologie, le nombre d'heures travaillées a augmenté de 59 \% depuis 1992 et la tendance continue. En cardiologie, il y a eu une augmentation des admissions de 9,48\% depuis 1993-1994. En santé mentale, l'augmentation des admissions est de 14,48 \% depuis 1993-1994, malgré un virage ambulatoire important. Dans les cas de maladies respiratoires et de chirurgie cardiaque, la consommation de services a augmenté respectivement de $3,83 \%$ et de $4,21 \%$ durant les deux dernières années. Les hausses ont été les plus marquées parmi le groupe des 75 à 79 ans ». (Association des hôpitaux du Québec, [Page consultée le 29 septembre 2002], "L'augmentation de la demande de services est responsable des difficultés budgétaires des hôpitaux», [En ligne], Adresse URL :

http://www.ahq.org/info/nouvelles/presse/c060700.html)

17. Voir, entre autres, les travaux suivants sur le paradoxe de productivité dans le cadre des services : Rallet Alain, «L'efficacité des technologies de l'information et de la communication à l'étape des réseaux ", dans Monnoyer-Longé Marie-Christine (sous la direction), L'entreprise et l'outil informationnel, Paris, Montréal, L'Harmattan, 1997 ; et Attewell Paul, «L'informatique et le paradoxe de productivité ", Le communicateur, no. 17, 1992, p. 1-27.

18. Il s'agit d'ailleurs d'une idée qui revient avec récurrence dans les documents officiels publiés par l'État québécois. Voir, entre autres, Ministère de la Santé et des services sociaux, Direction générale de l'administration et des immobilisations et Direction générale des ressources informationnelles, Prendre l'autoroute de l'information.. Les prémisses du plan d'action pour le secteur sociosanitaire, document de travail, Québec, MSSS, octobre 1995, p. 2, 6 et 17 ; Ministère de la Santé et des services sociaux, Direction des ressources informationnelles, Les orientations technologiques $d u$ réseau sociosanitaire, Orientations en télécommunication pour le secteur sociosanitaire, ouvrage cité, p. 5 et 25 ; et Ministère de la Santé et des services sociaux, L'autoroute de l'information, un levier essentiel à la transformation du système sociosanitaire - Plan d'action intégré, ouvrage cité, p. 34.

19. Gadrey Jean, L'économie des services, Paris, Éditions La Découverte, 1992.

20. Lacroix Jean-Guy, «Le sens sociopolitique de la façon de construire les autoroutes de l'information dans le secteur de la santé ", Actes du colloque Globalisme et pluralisme, Montréal, 24-27 avril 2002, Québec : Presses de l'Université Laval, 2003, p. 121 et suivantes.

21. Sicotte Claude, Champagne François et Contandriopoulos André-Pierre, «La performance organisationnelle des organismes publics de santé", Ruptures, revue transdisciplinaire en santé, vol. 6, no. 1, 1999, p. 37.

22. Contandriopoulos André-Pierre, "Économie du système de santé », dans Dufresne Jacques, Dumont Fernand et Martin Yves (dir.), Traité d'anthropologie médicale. L'institution de la santé et de la 
maladie, Sainte-Foy, Presses universitaire du Québec ; Québec, Institut québécois de recherche sur la culture ; Lyon, Presses universitaire de Lyon, 1985, p. 475.

\section{RÉSUMÉS}

Dans cet article, nous discutons du sens de l'implantation et du déploiement des technologies de l'information et de la communication (TIC) dans les organisations de soins au Québec, à la suite de la mise en place du virage ambulatoire informatisé qui s'impose comme la solution fondamentale, pour l'État et les décideurs, à l'objectif de réduction des coûts des services de soins assumés par le secteur public. Cette solution passe par une augmentation, intensification, importante du travail médical homologue à celle qui prévaut dans la production industrielle en économie capitaliste. Voilà ce que nous montrerons à la lumière d'extraits d'entretiens effectués sur le terrain.

In this paper, we discuss about the direction of the establishment and deployment of the information-communication technologies (ICT) in the healthcare organizations in Quebec, following the "virage ambulatoire informatisé" which was considered as the main solution, by the State and the decision makers, for reducing the public health care spendings. This solution means that we want to increase and intensify medical pratices similar to those observed in industrial mass production. This paper is based on an analysis of interviews with key informants selected on the basis of their involvement in reorganising the Quebec health care system around IT-based outpatient care, in order to show the relationship between the "virage ambulatoire informatisé" and the medical practices inside healthcare organizations.

\section{AUTEUR}

\section{LUC BONNEVILLE}

Luc Bonneville, après avoir complété une thèse de doctorat en sociologie à l'Université du Québec à Montréal, Luc Bonneville a mené en 2003-2004 des recherches post-doctorales au Groupe de recherche interdisciplinaire en santé de la Faculté de médecine de l'Université de Montréal. Il occupe maintenant un poste de professeur-chercheur au département de communication de l'Université d'Ottawa. Mail : luc.bonneville@uottawa.ca 